REVISTA CIENCIAS BIOMÉDICAS

ARTÍCULOS ORIGINALES

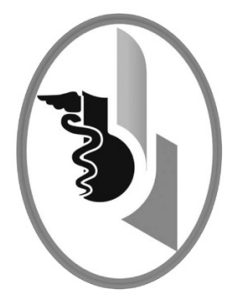

\title{
CARACTERIZACIÓN DE DENGUE Y DENGUE SEVERO EN ADULTOS HOSPITALIZADOS EN UNA INSTITUCIÓN DE CARTAGENA-COLOMBIA
}

\author{
DENGUE AND SEVERE DENGUE CHARACTERIZATION \\ IN INPATIENTS ADULTS OF AN INSTITUTION IN \\ CARTAGENA-COLOMBIA
}

Vergara-Verbel Benjamín Rafael ${ }^{1}$

Bello-Espinosa Ariel ${ }^{2}$

De la Vega-Del Risco Fernando ${ }^{3}$

Correspondencias: mdbenver@gmail.com

Recibido para evaluación: marzo - 20 - 2015. Aceptado para publicación: julio -20 - 2016

\section{RESUMEN}

Introducción: la infección por dengue es una de las arbovirosis de mayor frecuencia y morbimortalidad a nivel mundial, y de gran incidencia en el país. Colombia es el segundo país de América con mayor número de casos de muerte por dengue. La categorización de riesgo, de acuerdo a los criterios de la OMS, reconoce su relación con complicaciones en uno o más órganos durante el curso de la virosis. No hay estudios publicados en este medio que describan en la actualidad el curso de la infección en pacientes adultos ni que caractericen su curso de severidad.

Objetivo: caracterizar la frecuencia de aparición de dengue y sus complicaciones en pacientes adultos que acudieron a un centro de referencia en Cartagena (Colombia), durante el período comprendido entre enero de 2013 y enero de 2016, y reconocer aquellos con criterios de gravedad.

Materiales y métodos: estudio descriptivo, observacional, retrospectivo y transversal en pacientes mayores de 18 años con dengue y confirmación serológica, hospitalizados entre enero de 2013 y enero 2016 en la ESE Hospital Universitario del Caribe. Las fuentes de información fueron las historias clínicas del centro de estudio.

Resultados: se registraron para el estudio 95 casos de dengue, de los cuales 83 $(87.3 \%)$ fueron categorizados como dengue con signos de alarma y 12 casos $(12.6 \%)$ como dengue grave. Desde 2013, se observó una disminución progresiva en la incidencia del dengue en el centro estudiado. Las manifestaciones clínicas más frecuentes fueron fiebre, cefalea, mialgias y artralgias. Las alteraciones de laboratorio más comunes fueron trombocitopenia y la elevación de las aminotransferasas, principalmente la AST. Los signos ecográficos de colecistitis acalculosa fueron especialmente frecuentes. No se registraron casos fatales.

1 Médico. Estudiante de Postgrado. Medicina Interna. Facultad de Medicina. Universidad de Cartagena. Cartagena. Colombia.

2 Médico. Especialista en Medicina Interna. Docente Facultad de Medicina. Universidad de Cartagena. Cartagena. Colombia.

3 Médico. Especialista en Medicina Interna e Infectología. Docente Facultad de Medicina. Universidad de Cartagena. Cartagena. Colombia. 
Conclusión: la infección por dengue sigue siendo una causa frecuente de hospitalización en este medio, pero que muestra un comportamiento en descenso. Existe dificultad en la categorización de pacientes de acuerdo a los criterios de severidad de la OMS. La colecistitis acalculosa es un hallazgo frecuente y aún debe precisarse su rol en el seguimiento de los pacientes. Rev.cienc.biomed. 2016;7(2):212-222.

\section{PALABRAS CLAVE}

Dengue; Dengue grave; Colecistitis acalculosa.

\section{SUMMARY}

Introduction: dengue virus infection is one of the most frequent arboviruses that causes high morbidity and mortality worldwide, especially in our country. Colombia is the second country with the highest number of fatal dengue cases in America. The categorization of the patients' risk according to the WHO criteria illustrates the relationship with severity and complications in one or more organs during the course of the disease. There are not recent studies that describe the course of infection in this region in adult patients.

Objective: to describe the frequency and profile of dengue cases in a tertiary hospital in Cartagena (Colombia), during a follow up period between January 2013 and January 2016, and to recognize those who presented severity criteria.

Material and methods: a descriptive, observational, retrospective and transversal study was carried out with patients that presented dengue and were over 18 yearsold with serological confirmation. They were hospitalized between January 2013 and January 2016 in the ESE Hospital Universitario del Caribe. The source of data were the medical histories and records of the hospital.

Results: 95 cases confirmed dengue: 83 cases (87.3\%) were categorized as dengue with alarms signs and 12 cases $(12.6 \%)$ as severe dengue. There was a gradual reduction in the incidence of dengue following the first year (2013). Fever, headache, myalgia and arthralgia were the most frequent symptoms. The most common laboratory abnormalities were thrombocytopenia and elevation of aminotransferases, especially SGOT. Ultrasonographical findings were very common and compatible with acalculous cholecystitis. There were not deaths related to the disease in the evaluated period.

Conclusion: dengue infection remains a frequent cause of hospitalization in our region, but shows a tendency to reduce its incidence. There's still some difficulties to classify patients according to the WHO severity criteria. Acalculous cholecystitis was a frequent finding and its value during patient follow-up requires more attention. Rev. cienc.biomed. 2016;7(2):212-222.

\section{KEYWORDS}

Dengue; Severe Dengue; Acalculous cholecystitis.

\section{INTRODUCCIÓN}

El dengue es una enfermedad viral aguda, endemo-epidémica, causada por un arbovirus de la familia Flaviviridae y transmitida por la picadura de mosquitos hembra del género aedes [principalmente aegypti y albopictus] (1) que produce una alta carga de morbimortalidad y se constituye en un problema de salud pública en el país. En América, Colombia ocupa el segundo lugar en frecuencia de casos mortales (después de Brasil) y el primero en frecuencia de casos severos o "dengue grave", con una relación de 1 caso severo por cada 100 ocurridos $(1: 100)$, que corresponde a la tasa más alta de todo el cono andino (2). La mortalidad por dengue es evitable en más del $98 \%$ de los casos y depende principalmente de la detección precoz y el adecuado tratamiento de soporte (3).

Los entes en salud pública están destinando esfuerzos dentro del enfoque preventivo de la enfermedad, tomando medidas para la erradicación del vector y más recientemente, en formas de vacunación para inmunidad efectiva, las cuales han mostrado resultados preliminares muy positivos (4), sin embargo, el impacto de esta medida no se verá reflejado en el futuro próximo. Teniendo en cuenta que Cartagena es una ciudad costera rodeada de cuerpos de agua, con una población con limitaciones para el acceso a servicios de la 
salud, azotada además por otras arbovirosis como el zika y el chikungunya, virus que dificultan el diagnóstico diferencial. Se espera que el dengue siga comprometiendo la salud de sus habitantes en los años venideros.

En 2009 la Organización Mundial de la Salud (OMS), en un esfuerzo por incrementar la detección de casos potencialmente complicados y mejorar los protocolos de atención, sugirió nuevos criterios de clasificación de severidad $(5,6)$, catalogando como "dengue grave" aquellos casos en los que hubiera "daño severo a órganos", fuga capilar severa o hemorragia significativa. La presentación de estos casos graves genera costos directos e indirectos considerables (7). Desafortunadamente, la falta de definiciones claras para especificar algunas de estas categorías puede dar lugar a interpretaciones subjetivas por parte de los actores en salud, lo que acarrearía una falta de uniformidad en la atención de los casos. Esto es aún más preocupante si se reconoce que este medio carece de publicaciones oficiales que expresen la frecuencia en que estas formas severas están apareciendo o si el curso de la enfermedad está mostrando formas atípicas que escapan a esta clasificación propuesta.

La ESE Hospital Universitario del Caribe, ubicada en Cartagena, es una institución de referencia que recibe a gran número de pacientes remitidos desde el primer $y$ segundo nivel de atención, tanto del distrito como del departamento.

Ya que la información acerca de la epidemiología clínica de la enfermedad no debería surgir de países en donde la enfermedad por dengue no constituye un problema de salud pública, el desarrollo del conocimiento debe partir desde el proceder diario y de la investigación local y regional, motivados por la responsabilidad del aporte científico para los pacientes. En este sentido, se motiva la realización de este estudio que tiene como objetivo caracterizar los casos de pacientes adultos con dengue hospitalizados en el período de tiempo entre 2013-2015 e identificar aquellos casos que tuvieron curso severo de acuerdo a los criterios sugeridos por la OMS.

\section{MATERIALES Y MÉTODOS}

Se realizó estudio observacional, descriptivo, trasversal, en pacientes hospitalizados en la ESE Hospital Universitario del Caribe (Cartagena-Colombia), en el período comprendido entre el 1 de enero de 2013 hasta el 1 de enero de 2016, previa aprobación de su protocolo por las instituciones de investigación de la Universidad de Cartagena y de la Subgerencia Científica y de Proyección Social de la ESE. Para el estudio se consideraron elegibles a todos los pacientes con edad $\geq 18$ años que hubieran sido hospitalizados en el período de tiempo antes descrito por un cuadro febril compatible con dengue con serología confirmatoria IgM positiva. Entre estos, se excluyeron aquellas pacientes en estado de gestación, pacientes con serología positiva que fueron considerados "falsos positivos" por cuadros clínicos no concordantes, pacientes en quienes no fuera posible la obtención de la información médica o hubiesen sido remitidos a instituciones externas y sin que fuera posible su seguimiento.

Fuente de la información: historias clínicas y reportes de estudios complementarios que reposan en el archivo clínico de la Institución.

Búsqueda de la información: la búsqueda de pacientes se realizó mediante las codificaciones CIE-10 relacionadas con la enfermedad: A90X (fiebre del dengue 0 dengue clásico), A91X (fiebre del dengue hemorrágico), A928 (otras fiebres virales especificadas transmitidas por mosquitos) y A929 (fiebre viral transmitida por mosquito, sin otra especificación). Todos los pacientes que cumplieron las condiciones antes descritas fueron incluidos para la obtención de la información. Las variables a analizar fueron codificadas según la operacionalización definida en el protocolo y tabuladas en una base de datos generada en Microsoft Excel 2016 para posterior análisis estadístico.

Definiciones: para todo mayor de 18 años con cuadro compatible y serología IgM positiva se categorizó la enfermedad por dengue de acuerdo con los criterios de clasificación de la OMS (2009), así: 
Dengue con signos de alarma (DCSA): dolor abdominal, vómitos persistentes, acumulación de fluidos, sangrado de mucosas, letargia o inquietud, crecimiento hepático de más de $2 \mathrm{~cm}$, incremento en hematocrito junto con rápido descenso de plaquetas.

Dengue grave (DG): fuga de plasma severa que conlleve a shock o acumulación de fluidos con distrés respiratorio. Sangrado severo según "evaluación del clínico". Compromiso severo de órganos como el hígado (Aspartato aminotransferasa (AST) o Alanino aminotransferasa (ALT) $\geq 1.000$ ), sistema nervioso central (Ej., alteración del estado de conciencia), corazón u otros órganos.

Análisis estadístico: se realizó un análisis descriptivo, univariado, donde se determinaron las medidas de tendencia central para las variables cuantitativas con sus respectivas medidas de dispersión. Para las variables cualitativas se determinaron la frecuencia absoluta y relativa. Se realizó un análisis multivariado teniendo en cuenta las complicaciones más importantes, sin embargo, por el poco número de casos se restringió a un análisis descriptivo y no fue posible profundizar en análisis probabilístico.

\section{RESULTADOS}

Se incluyeron para este estudio un total de 95 pacientes con dengue, de los cuales 83 casos $(87.3 \%)$ fueron categorizados como DCSA y 12 casos (12.6\%) como DG. El $66.3 \%$ de los casos fueron hombres, con una razón hombre: mujer de 1.97. La media de edad fue de $29 \pm 13$ años. El $70.5 \%$ de los casos se hospitalizaron en el año 2013, con menor ocurrencia en los años subsiguientes. La mayoría de pacientes con DG $(66.7 \%)$ atendidos en la Institución fueron remitidos desde áreas rurales por fuera del distrito. Las características sociodemográficas se resumen en la Tabla No 1.

\begin{tabular}{|c|c|c|c|c|c|c|}
\hline \multicolumn{7}{|c|}{$\begin{array}{c}\text { TABLA No } 1 . \\
\text { VARIABLES SOCIODEMOGRÁFICAS }\end{array}$} \\
\hline & \multicolumn{2}{|c|}{$\begin{array}{l}\text { DENGUE TODOS } \\
\text { LOS CASOS }\end{array}$} & \multicolumn{2}{|c|}{$\begin{array}{l}\text { DENGUE CON } \\
\text { SIGNOS DE ALARMA }\end{array}$} & \multicolumn{2}{|c|}{$\begin{array}{l}\text { DENGUE } \\
\text { GRAVE }\end{array}$} \\
\hline & $\mathrm{n}=95$ & $\%$ & $\mathrm{n}=83$ & $\%$ & $\mathrm{n}=12$ & $\%$ \\
\hline \multicolumn{7}{|c|}{ Género } \\
\hline Masculino & 63 & 66.3 & 56 & 67.5 & 7 & 58.3 \\
\hline Femenino & 32 & 33.7 & 27 & 32.5 & 5 & 41.7 \\
\hline \multicolumn{7}{|c|}{ Procedencia } \\
\hline De la virgen y turística & 28 & 29.5 & 27 & 32.5 & 1 & 8.3 \\
\hline Fuera del distrito & 26 & 27.4 & 18 & 21.7 & 8 & 66.7 \\
\hline Histórica y del Caribe norte & 15 & 15.8 & 14 & 16.9 & 1 & 8.3 \\
\hline Industrial y de la bahía & 25 & 26.3 & 23 & 27.7 & 2 & 16.7 \\
\hline No descrita & 1 & 1.1 & 1 & 1.2 & 0 & 0.0 \\
\hline \multicolumn{7}{|c|}{ Año de hospitalización } \\
\hline Enero - diciembre 2013 & 67 & 70.5 & 62 & 92.5 & 5 & 7.5 \\
\hline Enero - diciembre 2014 & 16 & 16.8 & 14 & 87.5 & 2 & 12.5 \\
\hline Enero - diciembre 2015 & 12 & 12.6 & 7 & 58.3 & 5 & 41.6 \\
\hline \multicolumn{7}{|c|}{ Período de hospitalización } \\
\hline Trimestre enero-abril & 14 & 14.7 & 14 & 16.8 & 0 & 0 \\
\hline Trimestre mayo-agosto & 9 & 9.4 & 8 & 9.6 & 1 & 8.3 \\
\hline Trimestre septiembre-diciembre & 72 & 75.7 & 61 & 73.4 & 11 & 91.6 \\
\hline
\end{tabular}


Todos los pacientes cursaron con un síndrome febril. Las manifestaciones clínicas acompañantes más frecuentes fueron en su orden: cefalea, mialgias, artralgias y dolor abdominal (Tabla No 2).

Dentro del examen físico, el $26.3 \%$ de los pacientes tuvieron una prueba de torniquete positiva, $14.7 \%$ tuvo una prueba negativa $y$ en el $58.9 \%$ de los casos la prueba no fue especificada en la historia de ingreso a la Institución. El hallazgo de laboratorio más frecuente fue la trombocitopenia, que se presentó con un nadir de plaquetas de 66.168 en el grupo DCSA y de 17.166 en el grupo DG. Entre otros hallazgos paraclínicos se destaca la elevación de la creatin fosfokinasa (CPK) y de las aminotransferasas, con un mayor incremento de la AST sobre la ALT en una razón de 1.5:1. La prolongación del tiempo de tromboplastina activada fue frecuente en ambos grupos, pero fue superior en el DSCA (Tabla No 3).

Se les practicó radiografía de tórax a 58 pacientes $(61 \%)$, se encontró normal en el $93.1 \%$ de los casos. El hallazgo patológico más frecuente fue el derrame pleural en $6.8 \%$ de los casos. Se les practicó ecografía de abdomen total a 58 pacientes (61\%). En el grupo DCSA, los hallazgos más frecuentes fueron: ecografía normal $(38.7 \%)$, seguida de líquido libre en cavidad (36.7\%) y signos de colecistitis acalculosa (36.7\%). En el grupo DG, la ecografía abdominal fue normal en el $11.1 \%$ de los casos. Los hallazgos patológicos más frecuentes fueron: líquido libre en cavidad (66.6\%) y signos de colecistitis acalculosa (44.4\%) (Tabla No 3 ).

La estancia promedio de los pacientes fue de 4.4 días para el grupo DCSA y de 9 días para el grupo DG. En el grupo DCSA, el cristaloide más frecuentemente utilizado para la reanimación endovenosa fue la solución salina al $0.9 \%$ mientras que, en el grupo DG, la mayoría de pacientes fue manejado con lactato de Ringer. No se describió en ninguno de los casos el requerimiento de manejo con coloides o con aminas vasoactivas. En el grupo con DG se utilizaron hemoderivados en el $75 \%$ de los casos, mientras que en el grupo de DCSA en $4.8 \%$ de los casos. El hemoderivado de mayor uso fue el concentrado de plaquetas.

Se encontraron 12 casos de dengue considerados como dengue "grave" 0 "complicado", cuyas características se detallan en la Tabla No 4. De los 12 pacientes con complicaciones, 4 casos (33.3\%) fueron por fuga capilar severa, 6 casos (50\%) fueron por hemorragia "considerada por los tratantes como significativa" y 2 casos $(16.6 \%)$ por daño severo a órganos. No se registraron casos mortales de dengue por

\begin{tabular}{|c|c|c|c|c|c|c|}
\hline \multicolumn{7}{|c|}{ MANISFESTACIONES CLINICAS } \\
\hline & $\begin{array}{c}\text { DENGUE TODOS } \\
\text { LOS CASOS }\end{array}$ & $\begin{array}{c}\text { DENGUE CON SIGNOS } \\
\text { DE ALARMA }\end{array}$ & \multicolumn{2}{c|}{$\begin{array}{c}\text { DENGUE } \\
\text { GRAVE }\end{array}$} \\
\hline & $\mathrm{n}=95$ & $\%$ & $\mathrm{n}=83$ & $\%$ & $\mathrm{n}=12$ & $\%$ \\
\hline & 95 & 100 & 83 & 100 & 12 & 100 \\
\hline Fiebre & 70 & 73.7 & 62 & 74.7 & 8 & 66.7 \\
\hline Cefalea & 65 & 68.4 & 55 & 66.3 & 10 & 83.3 \\
\hline Mialgias & 65 & 68.4 & 56 & 67.5 & 9 & 75 \\
\hline Artralgias & 55 & 57.9 & 46 & 55.4 & 9 & 75 \\
\hline Dolor abdominal & 47 & 49.5 & 40 & 48.2 & 7 & 58.3 \\
\hline Náuseas y/o vómitos & 41 & 43.2 & 32 & 3.8 .6 & 9 & 75 \\
\hline Dolor retrocular & 22 & 23.2 & 17 & 20.5 & 5 & 41.7 \\
\hline Rash o exantema & 16 & 16.8 & 13 & 15.7 & 3 & 25 \\
\hline Diarrea & 3 & 3.2 & 2 & 2.4 & 1 & 8.3 \\
\hline Tos & 0 & 0.0 & 0 & 0.0 & 0 & 0.0 \\
\hline Odinofagia & 0 & 0.0 & 0 & 0.0 & 0 & 0.0 \\
\hline Disnea & & & & &
\end{tabular}




\begin{tabular}{|c|c|c|c|c|}
\hline \multicolumn{5}{|c|}{$\begin{array}{c}\text { TABLA N0 } 3 . \\
\text { HALLAZGOS MÁS RELEVANTES DE ESTUDIOS COMPLEMENTARIOS }\end{array}$} \\
\hline & \multicolumn{2}{|c|}{$\begin{array}{l}\text { DENGUE CON SIGNOS DE } \\
\text { ALARMA }\end{array}$} & \multicolumn{2}{|c|}{$\begin{array}{l}\text { DENGUE } \\
\text { GRAVE }\end{array}$} \\
\hline & $\mathrm{n}=83$ & $\%$ & $\mathrm{n}=12$ & $\%$ \\
\hline \multicolumn{5}{|c|}{ Laboratorios } \\
\hline Promedio de AST & 180.3 & - & 407.6 & - \\
\hline Promedio de ALT & 122.1 & - & 340.6 & - \\
\hline Promedio de nadir de plaquetas & 66.168 & - & 17.166 & - \\
\hline Promedio de CPK & 290.8 & - & 547.0 & - \\
\hline TTP prolongado & $62 / 68$ & 74.7 & $8 / 12$ & 66.7 \\
\hline \multicolumn{5}{|c|}{ Rayos $\mathrm{X}$ de tórax } \\
\hline Estudios realizados & 48 & 57.8 & 10 & 83.3 \\
\hline Estudio no realizado & 35 & 42.2 & 2 & 16.6 \\
\hline Estudios dentro de límites normales & 45 & 54.2 & 9 & 75 \\
\hline Derrame pleural (uni o bilateral) & 3 & 3.6 & 1 & 8.3 \\
\hline \multicolumn{5}{|c|}{ Ultrasonido de abdomen } \\
\hline Estudios realizados & 49 & 59 & 9 & 75 \\
\hline Estudio no realizado & 34 & 40.9 & 3 & 25 \\
\hline Estudios dentro de límites normales & 19 & 38.7 & 1 & 11.1 \\
\hline Líquido libre en cavidad & 18 & 36.7 & 6 & 66.6 \\
\hline Colecistitis alitiásica o hallazgos similares $t$ & 18 & 36.7 & 4 & 44.4 \\
\hline Hepato y/o esplenomegalia & 6 & 12.2 & 3 & 33.3 \\
\hline Otros procesos hepáticos $\neq$ & 4 & 8.1 & 0 & 0.0 \\
\hline Derrame pleural & 1 & 2 & 2 & 22.2 \\
\hline
\end{tabular}

* Un paciente puede presentar más de un hallazgo en los estudios de ecografía, por ejemplo, líquido libre y derrame pleural.

+ Colecistitis alitiásica, engrosamiento de vesícula biliar, edema perivesicular.

₹ Hígado graso, proceso inflamatorio hepático, entre otros.

\begin{tabular}{|l|c|c|c|c|c|c|}
\hline \multicolumn{7}{|c|}{ SERIE DE CASOS - DENGUE COMPLICADO } \\
\hline CASOS & SEXO & EDAD & $\begin{array}{c}\text { CRITERIOS POR } \\
\text { TRATANTES }\end{array}$ & $\begin{array}{c}\text { NADIR } \\
\text { PLAQUETAS }\end{array}$ & EVOLUCIÓN & MUERTE \\
\hline Caso 1 & Femenino & 18 & Sangrado & 21.000 & $\begin{array}{l}\text { Cuadro de 5 días con } \\
\text { hematemesis. }\end{array}$ & NO \\
\hline Caso 2 & Masculino & 19 & Sangrado & 26.000 & $\begin{array}{l}\text { Cuadro de 6 días con } \\
\text { gingivorragia persistente. }\end{array}$ & NO \\
\hline Caso 3 & Femenino & 20 & Acumulación & 12.000 & $\begin{array}{l}\text { Cuadro de 5 días de evolución } \\
\text { con gingivorragia persistente } \\
\text { de líquidos y acumulación de } \\
\text { líquido en cavidades. }\end{array}$ & NO \\
\hline Caso 4 & Masculino & 21 & Choque & $\begin{array}{l}\text { Cuadro de 4 días, signos de } \\
\text { shock distributivo ameritando } \\
\text { manejo en UCI con mejoría } \\
\text { con líquidos sin requerimiento } \\
\text { de vasopresores. }\end{array}$ & NO \\
\hline Caso 5 & Femenino & 22 & Sangrado & $\begin{array}{l}\text { Cuadro febril de 5 días de } \\
\text { evolución con gingivorragia } \\
\text { persistente. }\end{array}$ & No \\
\hline
\end{tabular}


Continua Tabla No 4.

\begin{tabular}{|c|c|c|c|c|c|c|}
\hline CASOS & SEXO & EDAD & $\begin{array}{l}\text { CRITERIOS POR } \\
\text { TRATANTES }\end{array}$ & $\begin{array}{c}\text { NADIR } \\
\text { PLAQUETAS }\end{array}$ & EVOLUCIÓN & MUERTE \\
\hline Caso 6 & Femenino & 25 & Choque & 7.000 & $\begin{array}{l}\text { Cuadro febril de } 5 \text { días con } \\
\text { sangrado gastrointestinal y } \\
\text { posteriores signos de shock. } \\
\text { Manejo con líquidos y soporte } \\
\text { transfusional. }\end{array}$ & NO \\
\hline Caso 7 & Masculino & 25 & $\begin{array}{l}\text { Acumulación de } \\
\text { líquidos }\end{array}$ & 23.000 & $\begin{array}{l}\text { Cuadro febril de } 7 \text { días con } \\
\text { derrame pleural, ascitis y de } \\
\text { líquidos dificultad respiratoria. }\end{array}$ & NO \\
\hline Caso 8 & Femenino & 32 & Sangrado & 13.000 & $\begin{array}{l}\text { Cuadro febril de } 4 \text { días con } \\
\text { sangrado por mucosas. } \\
\text { Serología IgM para leptospira } \\
\text { también positiva recibió } \\
\text { concomitante manejo } \\
\text { antibiótico. }\end{array}$ & NO \\
\hline Caso 9 & Masculino & 33 & Hepatitis & 35.000 & $\begin{array}{l}\text { Cuadro febril de } 4 \text { días con } \\
\text { trombocitopenia y elevación } \\
\text { de aminotransferasas (AST } \\
1.516 \mathrm{U} / \mathrm{mL} \text { y ALT } 1.441 \mathrm{U} / \\
\mathrm{mL} \text { ), sin deterioro neurológico } \\
\mathrm{ni} \text { criterios de falla hepática } \\
\text { fulminante, con recuperación } \\
\text { completa de las pruebas } \\
\text { hepáticas para el final de } \\
\text { su hospitalización. Pruebas } \\
\text { serológicas para otros } \\
\text { virus hepato-tropos fueron } \\
\text { negativas }\end{array}$ & NO \\
\hline Caso 10 & Masculino & 34 & Sangrado & 15.000 & $\begin{array}{l}\text { Cuadro febril de } 7 \text { días de } \\
\text { evolución con sangrado por } \\
\text { mucosas ameritando soporte } \\
\text { transfusional. }\end{array}$ & NO \\
\hline Caso 11 & Masculino & 36 & Sangrado & 6.000 & $\begin{array}{l}\text { Cuadro febril de } 5 \text { días de } \\
\text { evolución con trombocitopenia } \\
\text { profunda y rectorragia } \\
\text { ameritando soporte } \\
\text { transfusional. }\end{array}$ & NO \\
\hline Caso 12 & Masculino & 47 & Guillain-Barré & 2.000 & $\begin{array}{l}\text { Cuadro febril de } 14 \text { días de } \\
\text { evolución con trombocitopenia } \\
\text { profunda y persistente } \\
\text { acompañado de alteración } \\
\text { en la fuerza muscular en } \\
\text { miembros superiores e } \\
\text { inferiores, sin alteración } \\
\text { estructural en pruebas de TAC } \\
\text { y RNM de encéfalo, con clínica } \\
\text { y estudios de LCR compatibles } \\
\text { con síndrome de Guillain- } \\
\text { Barré. Su manejo incluyó } \\
\text { soporte con inmunoglobulina } \\
\text { endovenosa y también } \\
\text { con terapia antibiótica por } \\
\text { serología positiva para } \\
\text { leptospira (IgM). Su evolución } \\
\text { fue parcial hacia la mejoría, } \\
\text { aunque sin recuperación } \\
\text { completa de la fuerza } \\
\text { muscular. }\end{array}$ & NO \\
\hline
\end{tabular}


la unidad notificadora durante el período evaluado.

La Tabla N 5 presenta el promedio de algunas consideraciones clínicas, etarias y la estancia hospitalaria, según diversas complicaciones asociadas al dengue.

\section{DISCUSIÓN}

Este estudio demuestra que el dengue es una causa frecuente de hospitalización en el contexto colombiano, con una media de estancia hospitalaria significativa, mayor en el grupo de DG. Cabe anotar que se encontró una mayor incidencia de casos de dengue en 2013 (67 casos) y un número menor de casos en los años subsiguientes (16 casos en 2014 y 12 casos en 2015). Lo anterior puede corresponder a una disminución real en la incidencia de dengue o simplemente reflejar un descenso en el número de casos remitidos al centro estudiado, teniendo en cuenta además la posibilidad de sub-registro. Sin embargo, las publicaciones oficiales reportadas en el distrito de Cartagena con respecto a la notificación de casos de dengue también demuestran este fenómeno.
Así, en pacientes mayores de 15 años, se registraron en el distrito 523 casos de dengue en 2013 (8), luego en 2014 hubo una disminución hasta 369 casos (9), y finalmente en 2015, se registraron 213 casos (10). Hasta la semana epidemiológica 40 del 2016, se notificaron solo 72 casos, de los cuales ninguno presentó datos de gravedad (11). Lo anterior sugiere que, al menos en Colombia, la enfermedad está disminuyendo su incidencia. Esto contrasta con otras regiones del mundo, en donde la incidencia de dengue está incrementando notablemente con brotes epidemiológicos descritos desde 2014 en China (12), India (13), Malasia (14) y Taiwán (15).

En esta serie, las manifestaciones clínicas de dengue fueron variadas, pero son similares a las reportadas por otros estudios epidemiológicos $(16,17)$, describiendo igualmente qué suele presentarse como un cuadro febril con cefalea, mialgias y artralgias. Se ha propuesto que las manifestaciones clínicas pueden ser utilizadas para asistir en el diagnóstico diferencial con otras arbovirosis, de las cuales la artralgia suele ser menos frecuente en el dengue $y$

\begin{tabular}{|l|c|c|c|c|c|c|}
\hline \multicolumn{7}{|c|}{ TABLA NO 5. } \\
& Edad & $\begin{array}{c}\text { Estancia } \\
\text { hospitalaria }\end{array}$ & GPT & GOT & $\begin{array}{c}\text { Nadir } \\
\text { plaquetas }\end{array}$ & $\begin{array}{c}\text { Variación } \\
\text { del hema } \\
\text { tocrito }\end{array}$ \\
\hline & 27.6 & 9.08 & 340.60 & 407.60 & 17166 & -2.78 \\
\hline Dengue grave (N=12) & 29.7 & 4.4 & 122.18 & 180.37 & 66168 & -1.08 \\
\hline Dengue no grave (DCSA) & 47 & 36 & 42.00 & 37.00 & 2000 & -1.50 \\
\hline $\begin{array}{l}\text { Dengue con complicaciones } \\
\text { neurológicas (N=1) }\end{array}$ & 29.3 & 4.7 & 150.48 & 210.56 & 60595 & -1.30 \\
\hline $\begin{array}{l}\text { Dengue sin complicaciones } \\
\text { neurológicas }\end{array}$ & 22.7 & 7.2 & 309 & 375 & 14250 & -1.4 \\
\hline $\begin{array}{l}\text { Dengue con shock o } \\
\text { acumulación de fluidos (N=4) }\end{array}$ & 29.8 & 4.9 & 142.9 & 202 & 61989 & -1.3 \\
\hline Dengue sin shock & 33 & 8 & 1441.00 & 1516.00 & 35000 & -6.80 \\
\hline Dengue con hepatitis (N=1) & 29.4 & 5.0 & 133.00 & 192.08 & 60244 & -1.24 \\
\hline Dengue sin hepatitis & 26.8 & 6 & 199 & 279.6 & 18666 & -3.25 \\
\hline $\begin{array}{l}\text { Dengue con sangrado } \\
\text { relevante (N=6) }\end{array}$ & 29.6 & 4.9 & 145.8 & 203.7 & 62764 & -1.1 \\
\hline Dengue sin sangrado relevante & 27.7 & 4.9 & 183.2 & 278.7 & 33818 & -2.0 \\
\hline Dengue con colecistitis (N=22) & 33.3 & 5.64 & 112.4 & 156.1 & 59794 & 0.83 \\
\hline Dengue sin colecistitis & & & & & \\
\hline
\end{tabular}


más común en el Chikungunya virus (18), sin embargo, fue reconocida en el $68.4 \%$ de los casos. La prueba de torniquete es un signo clínico de buena sensibilidad en el abordaje del paciente con dengue, pero de acuerdo a lo encontrado parece estar subutilizada, por lo cual este estudio invita a una mayor educación respecto a esta prueba fácil de realizar en los centros de atención. Se reconoce en este estudio que las manifestaciones menos frecuentes fueron la diarrea, la odinofagia y los síntomas respiratorios. Lo anterior contrasta con un estudio en Antioquia (Colombia) en donde la tos, la anorexia y la astenia fueron los síntomas más frecuentes (19).

Dentro de las manifestaciones paraclínicas pudo observarse que la elevación de aminotransferasas fue mayor en el grupo de DG, e igualmente hubo un nadir de plaquetas más profundo. Es llamativa la elevación predominante de la AST sobre la ALT a diferencia de otras hepatitis por virus hepatotropos, lo cual parece estar en relación al daño muscular originado por el dengue, y de allí también el compromiso de la CPK. Esta predominancia de la AST ha sido descrita también en estudios previos (20).

Uno de los hallazgos más interesantes de esta investigación es la alta frecuencia en que se describieron los signos ultrasonográficos de colecistitis acalculosa aguda, la cual apareció en el $23.7 \%$ de todos los pacientes incluidos en el estudio, similar a la reportada por el estudio de Bhatty et al. (21). La guía colombiana de infectología para el manejo de dengue (22) y la Guía de práctica clínica colombiana para el manejo integral del dengue (23) reconocen a la colecistitis acalculosa como un criterio de dengue grave, mientras que la OMS en su publicación original para las Américas $(6,18)$ no lo hace. Otras publicaciones de la OMS sí reconocen la colecistitis acalculosa como predictor de desenlaces adversos.

De haberse utilizado este criterio, el número de pacientes con DG hubiera aumentado a 22 casos. Se observó que los pacientes que presentaron colecistitis acalculosa tuvieron mayor elevación de aminotransferasas y menor número de plaquetas comparados con aquellos que no la presentaron, sin embargo, la estancia hospitalaria fue menor que los otros casos de complicaciones. No es clara la fisiopatología de la colecistitis acalculosa. En este estudio, el 15\% de los casos tenían signos de colecistitis acalculosa sin que hubiera líquido libre aparente en cavidad peritoneal, en el $18 \%$ hubo líquido libre sin que existiera patología de vesícula biliar aparente, y en la mayoría de los casos $(67 \%)$ hubo ambos hallazgos. Se requieren más estudios para definir su rol en el seguimiento de los pacientes con dengue.

El manejo de soporte de los pacientes con líquidos endovenosos varió en los dos grupos, pero, se reconoce que, en todos los pacientes con DCSA, la estimación de líquidos fue acorde a lo planteado por la OMS, que recomienda un valor inicial sugerido de $5-7 \mathrm{cc} / \mathrm{kg} /$ peso (6). Puede suponerse que el adecuado manejo con líquidos endovenosos haya influido en el bajo número de casos con dengue grave encontrado. Para situaciones futuras a esta publicación, las recomendaciones más recientes de dengue para las Américas (2016) establecen para el grupo DCSA iniciar con una tasa de infusión de líquidos de $10 \mathrm{cc} / \mathrm{kg} /$ peso (18). El uso de hemoderivados fue frecuente en ambos grupos, aunque mucho mayor en DG. No se encontraron casos de muerte por dengue, incluso a pesar de haber identificado casos de DG con hemorragia gastrointestinal, la cual se ha descrito en otros estudios como un factor asociado a mortalidad (24).

Al igual que en el estudio de Gómez et al. (25), se encontró dificultad en realizar una adecuada categorización de los casos severos (DG) de acuerdo a los criterios recomendados. En muchos casos, la hemorragia considerada como significativa fue por sangrado de mucosas persistente sin que llevara al paciente a estado de choque ni a transfusión de glóbulos rojos. Por otra parte, se consideró como dengue grave un caso de síndrome de Guillain-Barré, el cual ha sido descrito como una forma atípica pero complicada del dengue $(26,27)$, sin que cumpla la definición de la OMS de compromiso grave de SNC. Es necesario dar claridad respecto a la definición de criterios de severidad para permitir uniformidad en la clasificación y manejo, así como un mayor 
reconocimiento y descripción de las formas atípicas en el medio.

Este estudio consiguió un número representativo de casos con criterios de inclusión amplios que permitieron evaluar a todos los pacientes que hubieran sido detectados con diagnósticos relacionados con la enfermedad. Los datos obtenidos permiten generar un nuevo conocimiento respecto al comportamiento del dengue y de sus formas severas en el contexto local, y las principales alteraciones paraclínicas e imagenológicas. Puede afirmarse que el descenso en el número de casos de dengue observado indica que se trata de una enfermedad que está teniendo menor impacto en la ciudad de Cartagena, al menos en términos de incidencia. Igualmente, la ausencia de casos mortales sugiere que se están reconociendo los casos de manera oportuna y ofreciendo un manejo adecuado. Adicionalmente, este estudio tiene como fortaleza que identifica cuáles son las principales dificultades en el abordaje, diagnóstico y clasificación de pacientes, permitiendo generar actitudes de cambio concretas con el objetivo de mejorar la atención.

Pese a ello, se reconoce igualmente que este estudio tiene varias debilidades. En primer lugar, se admite la naturaleza descriptiva y retrospectiva del estudio realizado, requiriendo además la exclusión de pacientes que pudieron haber presentado otro tipo de complicaciones (cardiovasculares, renales o de otros órganos), pero cuya información no estuvo disponible para su revisión por ser las historias clínicas la fuente de información. En ese sentido, también es importante resaltar la utilización exclusiva de la serología IgM como criterio serológico, ya que es el método paraclínico utilizado en el centro de atención, sin desconocer que el uso de otras herramientas serológicas como la proteína NS1 también hubiera permitido incluir otro número de pacientes. Se ha descrito, sin embargo, que la serología IgM es el método de diagnóstico más utilizado a nivel mundial (27).

A pesar de todo lo anterior, se considera que las debilidades metodológicas identificadas pueden estar sujetas a mejoras con la intención de producir estudios futuros que permitan una mejor caracterización de la enfermedad y mejorar los procesos de clasificación y manejo de los pacientes. El servicio médico debe procurar una atención oportuna y soporte apropiado para el manejo de los pacientes, pero también debe estar atento a formas atípicas e inusuales. Es recomendable proponer más estudios que permitan aclarar cuál es el rol de la colecistitis acalculosa como signo de gravedad.

\section{CONCLUSIÓN}

El dengue es causa frecuente de morbilidad en el medio. Categorizar a los pacientes con dengue grave permite reconocer un subgrupo de pacientes con hallazgos paraclínicos, imagenológicos y curso clínico más severo que posiblemente requieran mayores esfuerzos.

CONFLICTO DE INTERESES: ninguno que declarar.

FINANCIACIÓN: recursos propios de los autores.

\section{REFERENCIAS BIBLIOGRÁFICAS}

1. Simmons C, Farrar J, Nguyen vV, Wills B. Dengue. N Engl J Med. 2012;366(15): 1423-32.

2. OMS. Number of reported cases of dengue and severe dengue (SD) in the Americas, by country: figures for 2016 (to week noted by each country) Epidemiological Week / EW 41 (Updated October 28,2016). Disponible en: http://www.paho.org/hq/index.php?option=com_ docman\&task=doc_download\&Itemid=\&gid= 36681\&lang=es.

3. Ministerio de Protección Social (Col), Instituto Nacional de Salud; Organización Panamericana de la Salud. Guía de atención clínica integral del paciente con dengue. 2010.15 páginas. Disponible en: http://www.paho.org/col/index.php?option=com_docman\&task=doc_ download\&gid=432\&Item.

4. Villar L, Dayan GH, Arredondo-García JL, Rivera DM, Cunha R, Deseda C, et al. Efficacy of a tetravalent dengue vaccine in children in Latin America. N Engl J Med. 2015;372(2):113-23.

5. Lin C, Huang C, Chen Y. Classification of dengue: the clinical use of World Health Organization 2009 guideline. J Formos Med Assoc. 2013; 112(2):61-3. 
6. World Health Organization (WHO). Special programme for research and training in tropical diseases (TDR). Dengue: guidelines for diagnosis, treatment, prevention and control -- New edition. France: WHO Library Cataloguing-in-Publication Data. 2009 Aug [Citado 2014 May 09]. 160 páginas. Disponible en: http://www.who.int/tdr/publications/documents/denguediagnosis.pdf.

7. Castro-Rodríguez R, Galera-Gelvez K, López Yescas JG, Rueda-Gallardo JA. Costs of dengue to the health system and individuals in Colombia from 2010 to 2012. Am J Trop Med Hyg. 2015; 92(4):709-14.

8. Guerra C; Alcaldia Mayor de Cartagena de Indias. Comportamiento epidemiológico de dengue, distrito de Cartagena, Semana 48 de 2013. Boletín Epidemiológico. [Citado 2016 Nov14]. 5 páginas. Disponible en: http://dadiscartagena.gov.co/images/docs/saludpublica/ vigilancia/boletines/2013/dengue_has ta_sem_48.pdf

9. Guerra C; Alcaldia Mayor de Cartagena de Indias. Comportamiento epidemiológico de enfermedades transmitidas por vectores. Distrito de Cartagena, Semana 53 de 2014. BoletínEpidemiológico. [Citado 2016 Nov 14]. 21páginas. Disponible en: http://dadiscartagena. gov.co/images/docs/saludpublica/vigilancia/boletines/2014/etv_sem_53_2014.pdf

10. Guerra C, Alcaldia Mayor de Cartagena de Indias. Comportamiento Epidemiologico de Enfermedades Transmitidas por Vectores, Distrito de Cartagena, Semana 53 de 2015. Boletín Epidemiológico. [Citado 2016Nov14].12páginas. Disponible en: http://dadiscartagena.gov. co/images/docs/saludpublica/vigilancia/boletines/2015/etv_sem_52 2015.pdf

11. Guerra C, Alcaldia Mayor de Cartagena de Indias. Comportamiento Epidemiologico Hasta la semana epidemiológica \#42 de enfermedades transmitidas por vectores, distrito de Cartagena. Boletín Epidemiológico. [Citado 2016 Nov 14]. 12 páginas.Disponible en: http:// dadiscartagena.gov.co/images/docs/saludpublica/vigilancia/boletines/2016/etv_sem_42 2016.pdf

12. Chen B, Liu Q. Dengue fever in China. Lancet. 2015.25;385(9978):1621-2.

13. Dengue challenges India's health system. Lancet. 2015. 26;386(10000):1212.

14. Pang EL, Loh HS. Current perspectives on dengue episode in Malaysia. Asian Pac J Trop Med. 2016;9(4):395-401.

15. Wang SF, Wang $W H$, Chang $K$, Chen $Y H$, Tseng $S P$, Yen $C H$, et al. Severe dengue fever outbreak in Taiwan. Am J Trop Med Hyg. 2016;94(1):193-7.

16. Rodríguez-Salazar C, Recalde-Reyes D, González M, Padilla Sanabria L, Quintero-Álvarez L, Gallego-Gómez J, et al. Clinical manifestations and laboratory findings on a case series of acute febrile syndrome with a presumptive diagnosis of dengue virus infection. Quindio, Colombia. Infectio. 2016;20(2):84-92.

17. Matta L, Barbosa M, Morales-Plaza C. Caracterización clínica de pacientes que consultaron por dengue en un hospital de tercer nivel en Cali, Colombia, 2013. Biomédica. 2016;36:133-9.

18. Organización Panamericana de la Salud. Dengue. Guías para la atención de enfermos en la región de las Americas 2 edición. Washington DC. 2016. [Citado 14 Nov 2016]. 144 páginas. Disponible en: http://www.who.int/tdr/publications/documents/dengue-diagnosis.pdf.

19. Restrepo BN, Beatty ME, Goez Y, Ramirez RE, Letson GW, Diaz FJ, et al. Frequency and clinical manifestations of dengue in urban medellin, Colombia. J Trop Med. 2014;2014:872608.

20. Wang XJ, Wei HX, Jiang SC, He C, Xu XJ, Peng HJ. Evaluation of aminotransferase abnormality in dengue patients: A meta-analysis. Acta Trop. 2016; 156:130-6.

21. Bhatty S, Shaikh NA, Fatima M, Sumbhuani AK. Acute acalculous cholecystitis in dengue fever. J Pak Med Assoc. 2009;59(8):519-21.

22. Malagon J, Padilla J, Rojas-Alvarez D. Guía de atención clínica integral del paciente con dengue. Infectio. 2011;15(4):1-9.

23. Ministerio de la Protección Social Colombia, Organización Panamericana de Salud. Guía para la atención clínica integral del paciente con dengue. [Citado 2016 Nov 14]. 46 páginas. Disponible en: https://www.minsalud.gov.co/Documentos\%20y\%20Publicaciones/ Gu\%C3\%ADa\%20para\%20la\%20atenci\%C3\%B3n\%20cl\%C3\%ADnica\%20integral\%20 del\%20paciente\%20con\%20de ngue.pdf

24. Pinto RC, Castro DB, Albuquerque BC, Sampaio Vde S, Passos RA, Costa CF, et al. Mortality predictors in patients with severe dengue in the state of Amazonas, Brazil. PLoS One. 2016;11(8): e0161884.

25. Gómez D, Causil C, Pinzón H, Suárez A, Moneriz C. Caracterización clínica del dengue en un hospital infantil de Cartagena (Colombia). Revista Científica Salud Uninorte. 2014;30(3):281-92.

26. Verma R, Sahu R, Holla V. Neurological manifestations of dengue infection: a review. J Neurol Sci. 2014;346(1-2):26-34.

27. Simon O, Billot S, Guyon D, Daures M, Descloux E, Gourinat AC, et al. Early Guillain-Barré Syndrome associated with acute dengue fever. J Clin Virol. 2016; 77:29-31.

28. Guzman MG, Harris E. Dengue. Lancet. 2015 Jan 31;385(9966):453-65. 